\title{
Optimization of an optrode microdevice for infrared neural stimulation
}

\author{
Ö.C. Boros ${ }^{1, *}$, Á.C. Horváth ${ }^{2,3,4}$, S. BelezNAI ${ }^{1}$, Ö. SEPsI ${ }^{1}$, D. Csősz ${ }^{1}$, Z. FeKETE \\ 2,3, P. KOPPA $^{1}$ \\ ${ }^{1}$ Department of Atomic Physics, Budapest University of Technology \& Economics, Budapest, Hungary \\ ${ }^{2}$ Research Group for Implantable Microsystems, Faculty of Information Technology \& Bionics, Pázmány Péter Catholic University, Budapest, \\ Hungary \\ ${ }^{3}$ Institute for Technical Physics \& Material Science, Centre for Energy Research, Hungarian Academy of Science, Budapest, Hungary \\ ${ }^{4}$ Óbuda University Doctoral School on Materials Sciences and Technologies, Budapest, Hungary \\ *Corresponding author: boroscsanadors@gmail.com
}

Received XX Month XXXX; revised XX Month, XXXX; accepted XX Month XXXX; posted XX Month XXXX (Doc. ID XXXXX); published XX Month XXXX

Infrared light is a promising candidate for the treatment of neurodegenerative diseases. Optimizing the device parameters to achieve the best optical and mechanical performance is essential for reliable in vivo operation. In this work, mechanical strength simulations and coupled optical and thermal model were used to determine optimal design parameters for maximizing overall device efficiency. Our analysis reveals that minimizing the number of integrated optical elements and optimizing of the optical path leads to $33 \%$ relative in-coupling efficiency improvement at equal mechanical robustness. Using a symmetric optrode tip with an angle of $15^{\circ}$, the efficiency showed further $17 \%$ relative improvement due to the enhancement of out-coupling at the tip. To investigate the temperature rise of the brain tissue during the infrared stimulation in the case of the optimized device, a thermal simulation with pulsed infrared excitation was developed. Our results show that the optimized device provides a temperature rise of $4.42^{\circ} \mathrm{C}$ as opposed to $3^{\circ} \mathrm{C}$ for the original setup.

(C) 2018 Optical Society of America

OCIS codes: (050.1940) Diffraction; (080.0080) Geometric optics; (290.1350) Backscattering; (290.4210) Multiple scattering.

http://dx.doi.org/10.1364/AO.99.099999

\section{INTRODUCTION}

Infrared neural stimulation (INS) is a technique that modulates the generation of action potential of neurons through temperature gradient induced by infrared light delivered to the tissue [1]. The radiant exposure has a maximum level, where action potentials are evoked without damage [2]. INS is superior to electrical stimulation since it avoids electrical interference and provides better spatial control [3]. The excited volume of neural tissue should be precisely determined for any stimulation device, therefore a reliable and validated numerical model is an efficient tool to predict the effect of design parameters on the device.

In our latest work, we introduced the design and fabrication of an implantable optrode microdevice [4], and developed its coupled optical and thermal model validated by optical measurements [5]. The model incorporated ray tracing to simulate the optical propagation in the device, Fourier scattering $[6,7]$ to describe the scattering on the optrode boundary and a calculation of the temperature distribution of the excited brain tissue as a consequence of the absorbed light intensity. Our model provides the flexibility to change all model parameters and therefore it is suitable for further optimization of the device.

A photo from the original chip-scale silicon optrode can be seen on Fig.1. The device is connected to the light source by an optical connector denoted by (a). Infrared light is coupled into the optrode (e) through a multimode optical fiber (b) placed to the tunnel (f). Light emitted from the fiber is coupled into the shaft (h) by a lens (g). The shaft is inserted in the brain tissue and infrared light is coupled out from the tip (i). The electrical signal of the brain tissue can be measured during stimulation by sensors integrated on the optrode surface [4]. The electrical signal is transferred by a printed circuit board (d) and an electrical connector (c). 
(A)

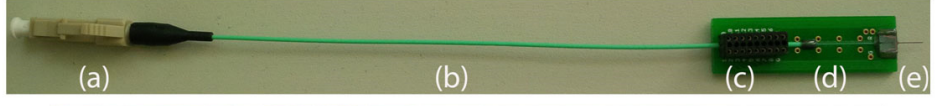

(B)

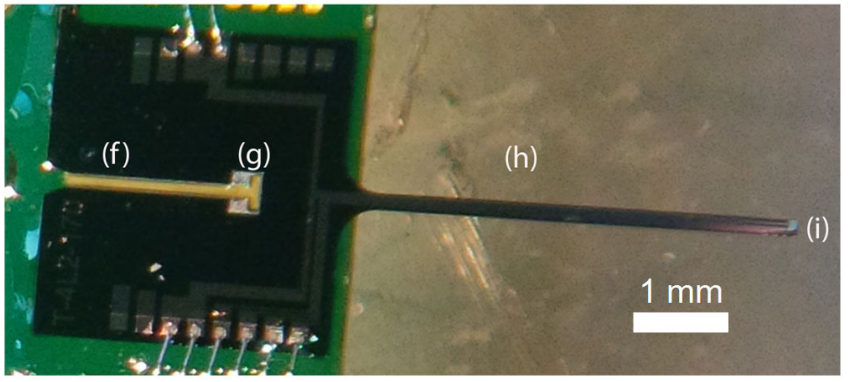

Fig.1. Schematic layout of a silicon optrode device (A) with a detailed view of the fiber output and focusing lens (B). (a) optical connector (b) multimode optical fiber (c) electrical connector (d) printed circuit board (e) optrode (f) fiber guide (g) lens (h) shaft (i) tip.

According to the simulations the overall efficiency of the device (defined as the ratio of the power delivered to the brain tissue and the output power from the fiber) was $30.5 \pm 2.5 \%$, which was in good agreement with the measurements. Using an excitation of $30 \mathrm{~mW}$ total power and $100 \mathrm{~ms}$ pulse width, the size of the excited volume was about $1 \mathrm{~mm}^{3}$ and the value of the maximal temperature rise of about $3^{\circ} \mathrm{C}$ occurred within 0.1- $0.2 \mathrm{~mm}$ from the tip [5].

In this paper, the optimization procedure of the optrode device is discussed. Mechanical, optical and thermal simulations were developed to investigate the mechanical robustness, the effect of tip shape, the absence of the lens and the length of the fiber guide on the overall efficiency of the device and on the thermal excitation. Our goal was to develop the optimized optrode design which produces higher overall device efficiency and a well localized thermal excitation in the brain tissue.

\section{SIMULATIONS}

The optimization of the device is based on our previous work [5] and is supplemented with mechanical modeling to investigate the mechanical strength of the device. We have calculated the von Mises stress in the device using a finite element model (FEM) developed in COMSOL $^{\circledR}$ Multiphysics - Solid Mechanics module [12] (see Fig.2).

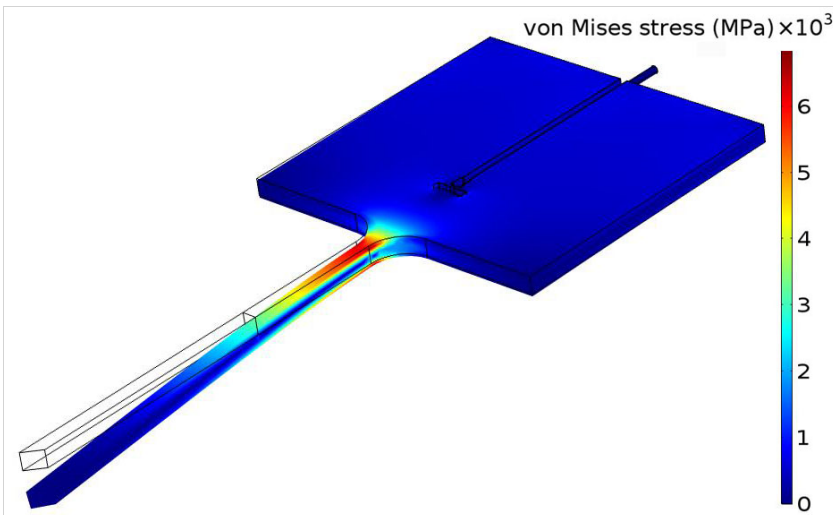

Fig. 2. Mechanical finite element model of the silicon optrode

The linear elastic material model was used for the calculation [13]. The stiffness tensor of silicon was used from the literature [14] according to the crystal orientation (110) of the microprobe. The shaft is inserted $3 \mathrm{~mm}$ deep into the brain tissue therefore the model uses a boundary load at this area perpendicular to the device top surface. The back of the optrode was set to fixed constraint. The input of the model was the total loading force and the output was the maximum of the von Mises stress in the device. The loading force was gradually increased until the maximum of the von Mises stress reached the yield strength of the silicon [15].

The overall device efficiency was calculated using a ray tracing model developed in Zemax ${ }^{\circledR}$ optical ray tracing software [16]. The excitation wavelength was $1310 \mathrm{~nm}$. According to our previous simulations the main losses occurred due to the reflections and due to the scattering on the material boundaries of the in-coupling and out-coupling interfaces of the device [5]. The in-coupling loss can be reduced using index matching glue between the fiber end and the optrode. The out-coupling loss can be optimized with shaping the tip of the optrode. The output of the optical model was the overall device efficiency values in air as well as the absorbed intensity distribution in the brain tissue.

To specify the heating effect during NIR stimulation a finite element thermal model was developed [5]. The model uses the absorbed light intensity calculated by the ray tracing model as heat source modulated by a periodic top-hat pulse with pulse-width of $100 \mathrm{~ms}$ and period of $500 \mathrm{~ms}$. The goal of the simulation was to estimate the temperature distribution of the brain tissue due to the NIR stimulation.

\section{RESULTS AND DISCUSSION}

\section{A. Optimization of the in-coupling components of the optrode}

Our previous calculations showed that the device has two important features which influence the loss at in-coupling. One of them is the distance between the in-coupling surface and the shaft, the second is the presence or the absence of the in-coupling lens. Therefore the effect of the geometry was investigated with and without lens as a function of the fiber guide length, hereafter denoted by $\ell$.

We have plotted the results of the mechanical and optical model (maximum load force and overall device efficiency) in Fig.3. The data are depicted as a function of $\ell$. Position A belongs to the original setup $\ell=2.33 \mathrm{~mm}$. When increasing $\ell$ the maximum load force has a steep decrease after point $B$ at $\ell=2.80 \mathrm{~mm}$ (white circles). Eliminating the lens the maximum load force remains at the initial value until position $C$ at $\ell=3.27 \mathrm{~mm}$ (black circles). After this position the maximum load force has a steep decrease. The overall device efficiency values given by the optical model show an increasing tendency with increasing $\ell$ (see Fig.3. (B)). The focusing property of the lens proved to be more and more negligible with increasing $\ell$. Summarizing the consequences, position $\mathrm{C}$ provided the optimized fiber end position regarding the mechanical strength and the optical overall device efficiency. The necessary fiber guide 


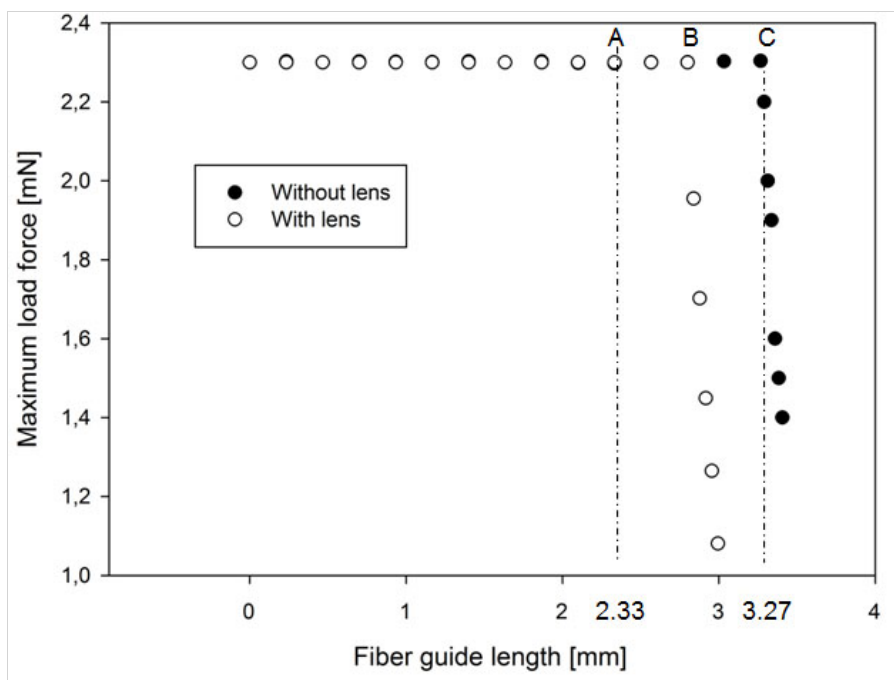

(A)

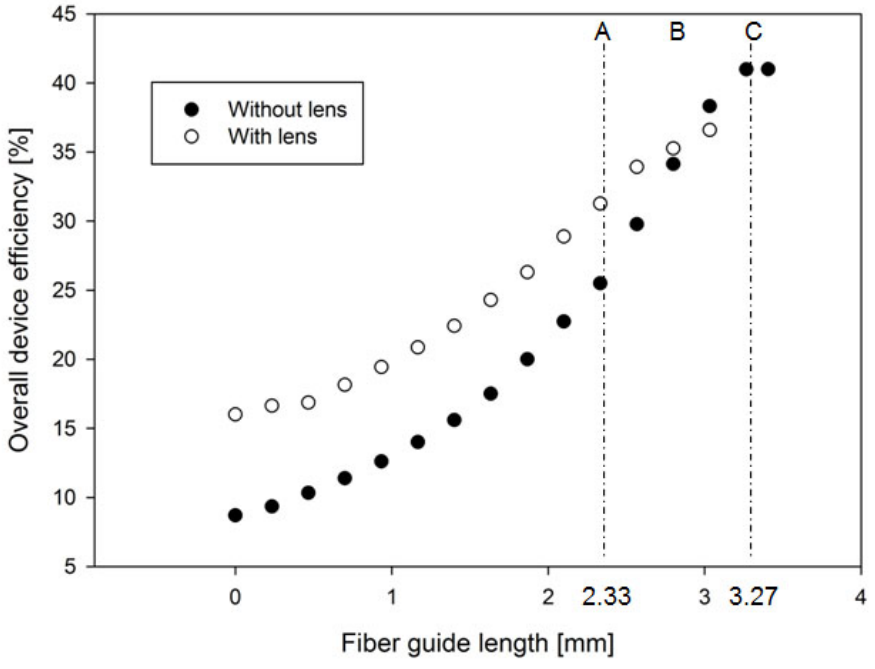

(B)

Fig.3. Results of the mechanical (A) and optical (B) simulations. The maximum load force and the overall device efficiency are shown depending on the fiber guide length $(\ell)$. Point A corresponds to the original $\ell=2.33 \mathrm{~mm}$, B to the mechanical threshold $\ell=2.80 \mathrm{~mm}$ with lens and $\mathrm{C}$ to the optimized $\ell=3.27 \mathrm{~mm}$ value without lens. The optimal fiber length elongation is $\Delta \ell=0.94 \mathrm{~mm}$.

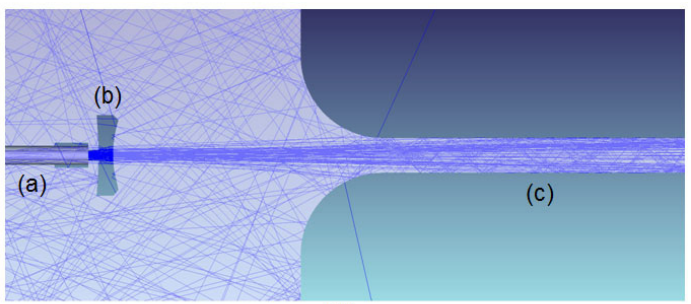

(A)

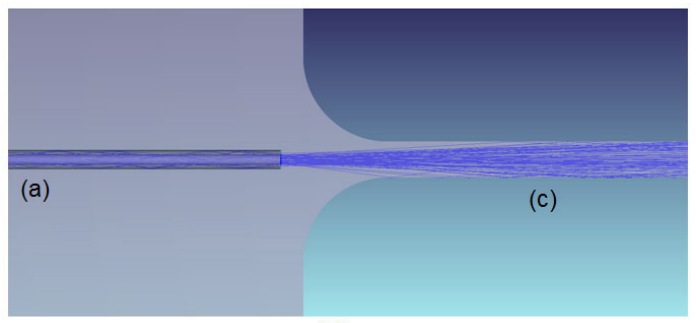

(B)

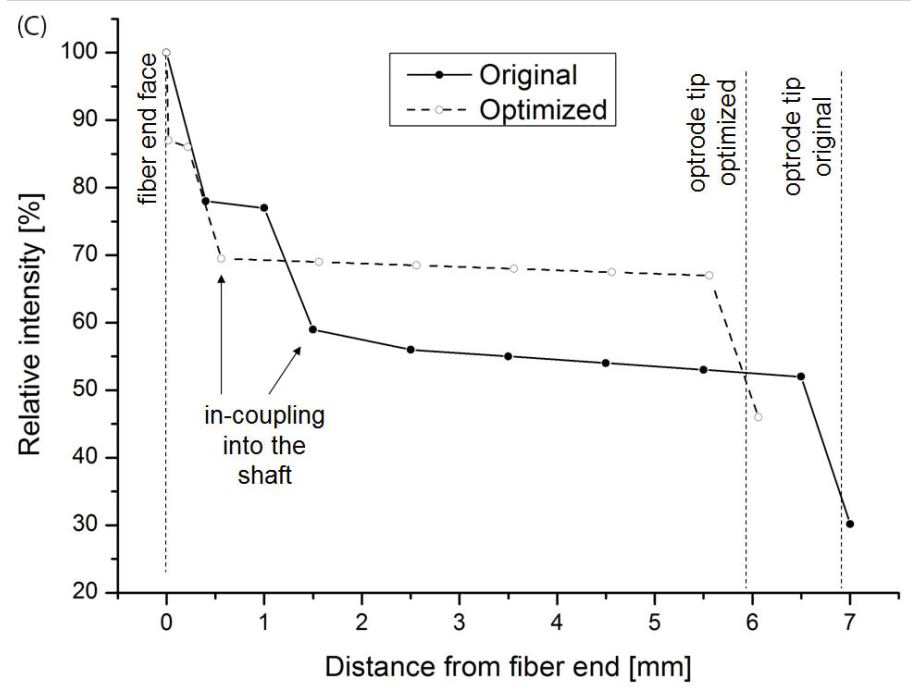

Distance from fiber end [mm]

Fig.4. (A) Schematic layout of the original and (B) optimized silicon optrode device (C) Relative intensity drop along the optrode length in the original and optimized setup. The parts of the device are: (a) fiber guide (b) focusing lens (c) shaft

elongation from original position is $\Delta \ell=0.94 \mathrm{~mm}$. At position $\mathrm{C}$ the relative intensity drop along the optrode length was determined and compared with the original setup [5] as it is shown in Fig.4. It can be observed that this setup provided significantly less in-coupling loss. In summary, eliminating the lens and elongating the fiber guide length by $0.94 \mathrm{~mm}$ increased the original $30.5 \pm 2.5 \%$ overall device efficiency to $40.1 \pm 2 \%$, using 4 different measured optrode surfaces to model scattered light [5].

\section{B. Optimization of the shaft tip}

A significant drawback of the original setup was that the rectangularshape tip generated high reflection loss and increased penetration force into the brain tissue [17]. To reduce these effects, symmetric and asymmetric sharp tip models of various angles of inclinations were developed and investigated. Fig.5. shows a simple analytic description for parallel rays in the vicinity of the tip. According to the simple geometric optical calculation in case of asymmetric tip angle $\alpha$ below $45^{\circ}$ and in case of symmetric tip angle $\alpha$ below $30^{\circ}$, the rays propagate trough total internal reflections towards the tip where the light will exit the device according the Fresnel equations (see Fig.5. (A) and (C)). Between $45^{\circ}$ and $53.35^{\circ}$ in case of asymmetric tip angle and between $30^{\circ}$ and $35.5^{\circ}$ in case of symmetric tip angle the most of the light power will exit the tip at point $\mathrm{P}_{2}$. In case of asymmetric tip in range of $53.35^{\circ} \leq \alpha \leq 73.35^{\circ}$ and in case of symmetric tip in range of $35.5^{\circ} \leq \alpha \leq 73.35^{\circ}$, light confinement can be observed due to total internal reflection (see Fig.5. (B) and (D)). Above tip angle $73.35^{\circ}$ the most of the light power will exit at point $P_{1}$.

In order to take the realistic angle distribution of the rays into account during optimization we have used the ray tracing model. The detectors measuring the output intensity of the tip were placed around the tip $0.375 \mathrm{~mm}$ far from the optrode surface and had 0.36 $\mathrm{mm} \times 0.36 \mathrm{~mm}$ size to involve all of the output intensity. The tip angle $\alpha$ was swept from $7^{\circ}$ to $90^{\circ}$. 

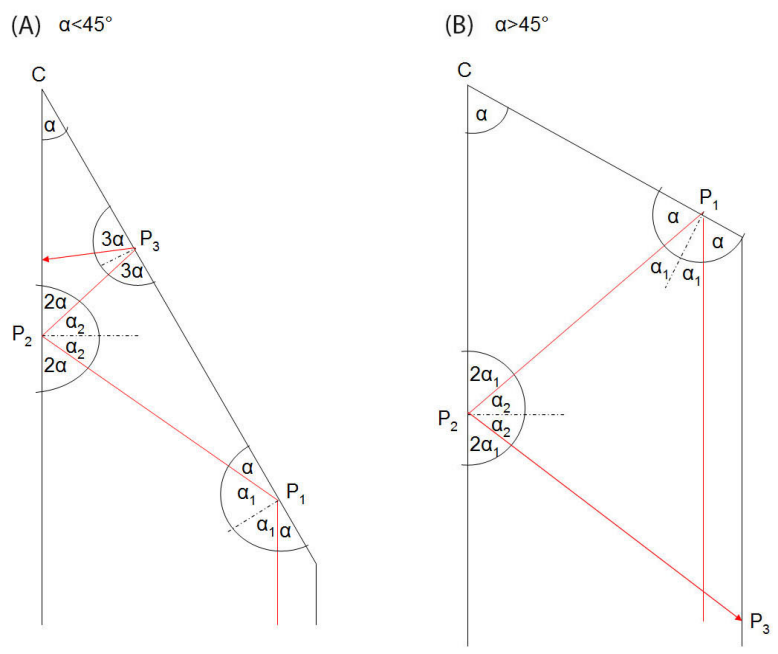

(C) $\alpha<30^{\circ}$

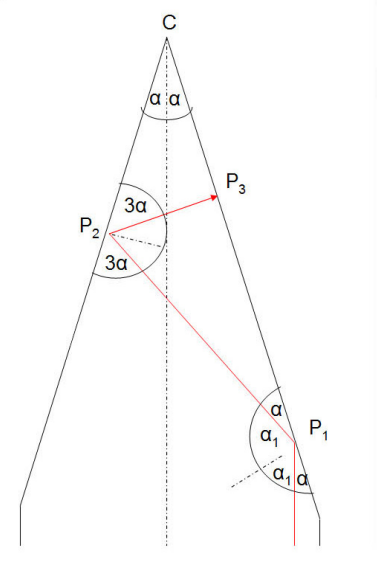

(D) $a>30^{\circ}$

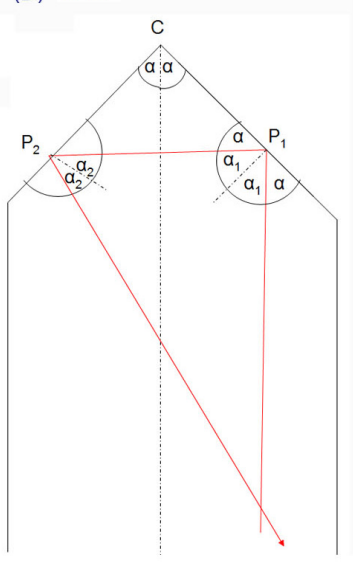

Fig.5. Sketches for the analytic description of the behaviors of parallel rays near the optrode tip (A) asymmetric tip with angle $\alpha$ below $45^{\circ}$ (B) asymmetric tip with angle $\alpha$ above $45^{\circ}$ (C) symmetric tip with angle $\alpha$ below $30^{\circ}$ (D) symmetric tip with angle $\alpha$ above $30^{\circ}$. Incident points and angles of the rays are denoted by $P_{k}$ and $\alpha_{k}$, respectively.

The real optrode geometry has not ideally sharp tip but has few micron fillet at the tip, which is an inherent feature of the fabrication technology relying on a final wet chemical polishing step [18]. Fig.6. shows a top view of the measured optrode tip using scanning electron microscope with the size of the fillet radius. The average radius of the fillet calculated from 4 different measured optrode profiles was $2.5 \mu \mathrm{m}$.

The overall device efficiency in case of elongated fiber guide setup depending on the tip angle is shown on Fig.7. (A), for ideally sharp tip and for a tip with a fillet of $2.5 \mu \mathrm{m}$. The simulation results show good agreement with the analytic approximation which predicted the possibility of total internal light confinement between $53.35^{\circ}$ and $73.35^{\circ}$ in asymmetric tip, and between $35.5^{\circ}$ and $73.35^{\circ}$ in symmetric tip setup. After this drop range the efficiency increased to the $40 \%$ value. The fillet of the tip led to a light efficiency decrease. It can be observed, that the effect of the fillet is more significant outside this drop range. In accordance with the analytic exceptions (see description of Fig.5.) decreasing the tip angle led to an increase in the efficiency. It should be noted that the penetration force and the mechanical stability during device implantation are also influenced by the tip angle [17], therefore in our setup we applied $15^{\circ}$ wedge tip for further simulations, which induces low penetration force during insertion, and acceptable mechanical robustness.

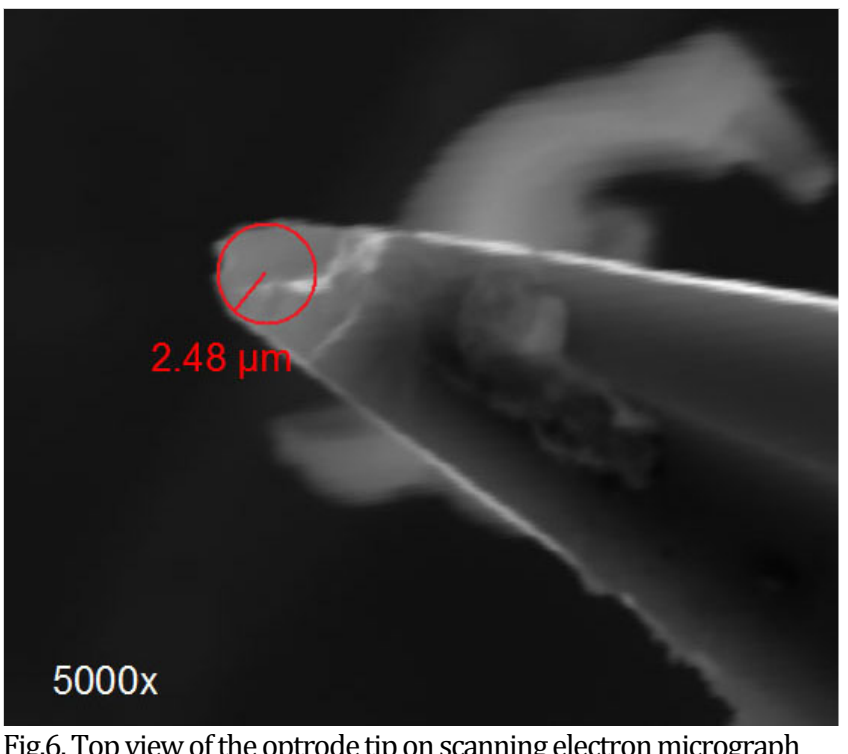

Fig.6. Top view of the optrode tip on scanning electron micrograph

The $15^{\circ}$ angle asymmetric and symmetric tip setups were used in the thermal model with pulsed excitation of $30 \mathrm{~mW}$ peak optical power and $100 \mathrm{~ms}$ pulse width. The results of the maximal temperature change at $\mathrm{t}=90 \mathrm{~ms}$ can be seen on Fig.7. (B). The maximal temperature change show significant correlation with the overall device efficiency. The asymmetric tip led to better localized excitation area, therefore the maximal temperature change is higher than in the symmetric tip setup. To investigate the effect of the fillet, simulations with $2.5 \mu \mathrm{m}$ fillets were run. The fillet of the tip led to a decrease the maximal temperature change with some tenth of a degree. Its reason is that according to the analytic theory, most of the light intensity exits the optrode in the vicinity of the tip. The maps of the light power density distribution emitted from the device and the temperature distribution around the tip at $\mathrm{t}=90 \mathrm{~ms}$ are shown in Fig.8. The temperature reaches its maximum value at around $0.1 \mathrm{~mm}$ distance from the optrode and $0.1 \mathrm{~s}$ after the start of the pulse. Maximal temperatures of the rectangular shape, asymmetric and symmetric tip setup with $2.5 \mu \mathrm{m}$ fillets are $4{ }^{\circ} \mathrm{C}, 4.42^{\circ} \mathrm{C}$ and $4.03^{\circ} \mathrm{C}$, respectively, instead of $3{ }^{\circ} \mathrm{C}$ belonging to the original setup [5]. The maximal temperature rise of the shaft is only $0.1^{\circ} \mathrm{C}$ due to the low absorption of silicon at the given wavelength and high heat conductivity of silicon compared to the water environment. Switching off the pulse led to a complete relaxation of the temperature in $0.4 \mathrm{~s}$ because the silicon shaft conducts the heat from the medium to the external environment. 


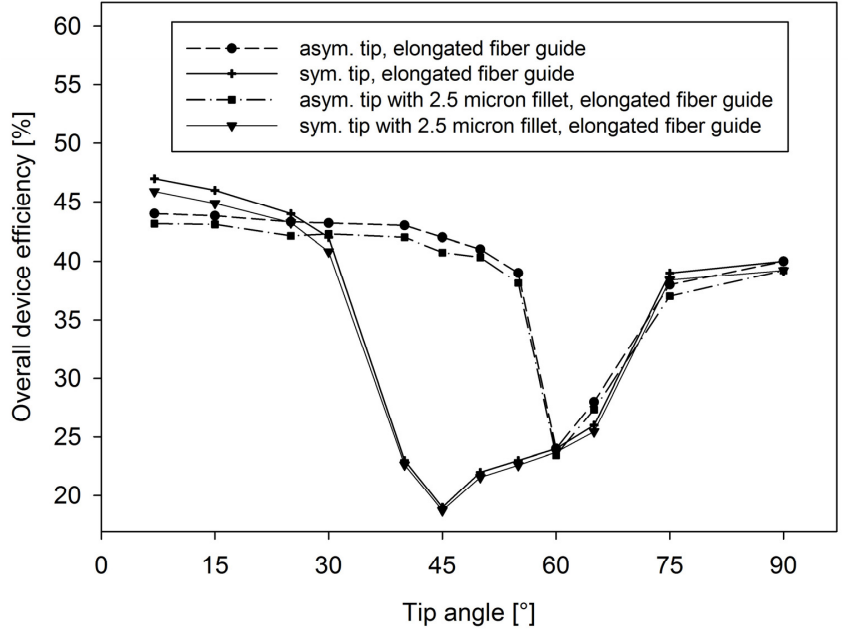

(A)

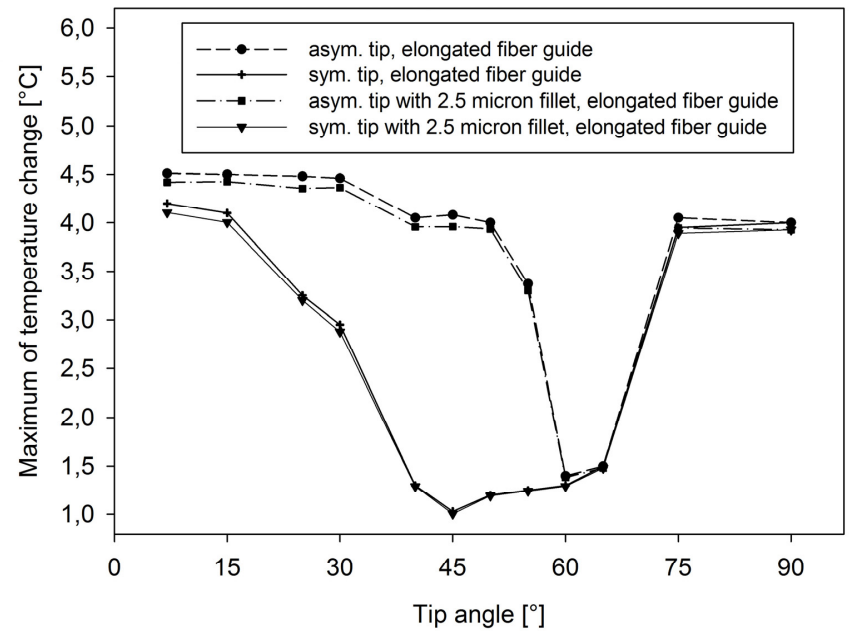

(B)

Fig.7. (A) The simulated overall device efficiency and (B) the simulated maximal temperature change plotted against the tip angle. Asymmetric tip is referred as asym. tip, symmetric tip is referred as sym. tip., with and without fillet.
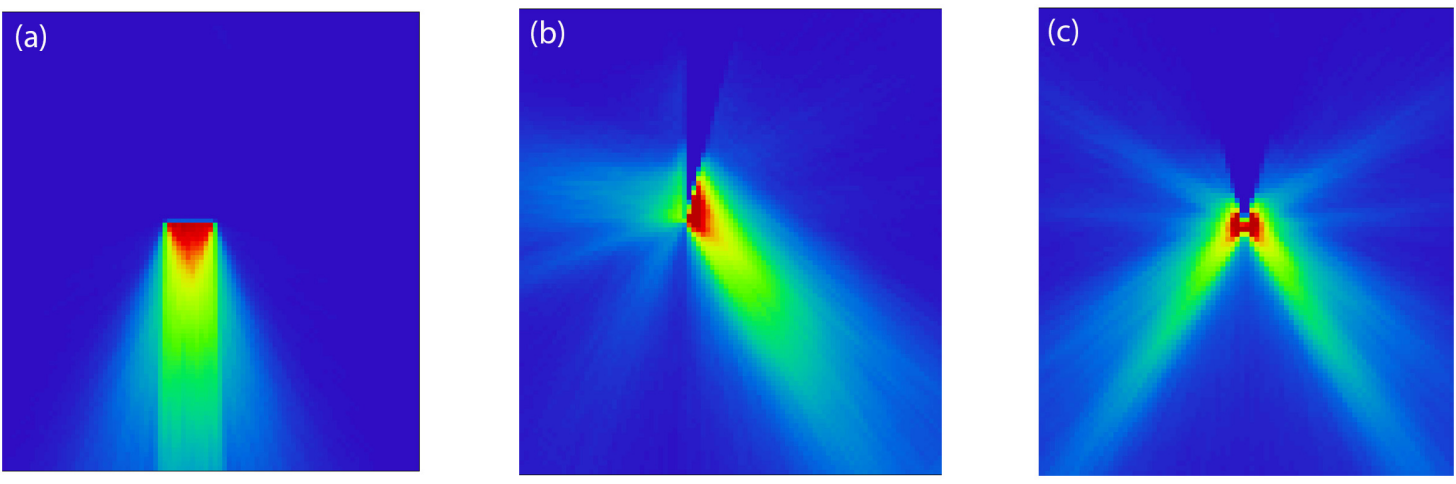

Power density $[\mathrm{W} / \mathrm{m} \wedge 2]$
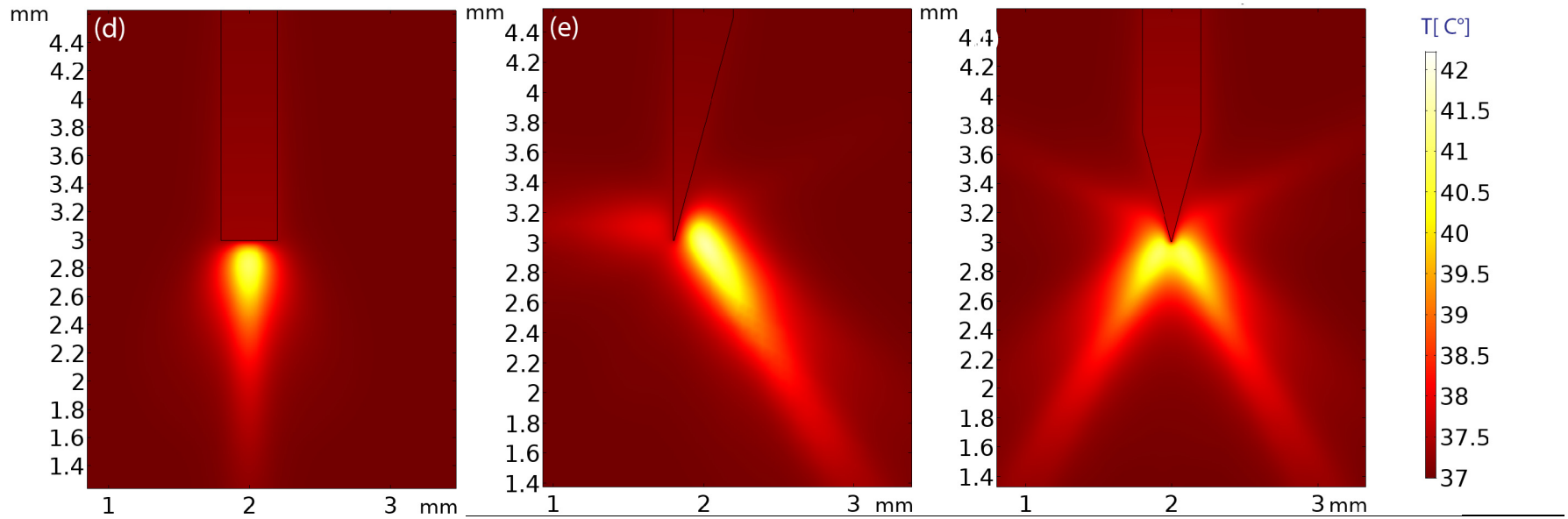

Fig.8. Maps of the light power density distribution emitted from the device (a) in case of the rectangular tip setup (b) asymmetric tip setup (c) symmetric tip setup with $2.5 \mu$ m radius of tip fillet. Temperature distributions using excitation with $30 \mathrm{~mW}$ total power, $100 \mathrm{~ms}$ pulse width at $\mathrm{t}=90$ $\mathrm{ms}$ (d) the excited area in case of the rectangular tip setup (e) asymmetric tip setup (f) symmetric tip setup with $2.5 \mu \mathrm{m}$ radius of tip fillet. Maximum temperatures are $4^{\circ} \mathrm{C}, 4.42^{\circ} \mathrm{C}$ and $4.03^{\circ} \mathrm{C}$, respectively.

We have carried out a measurement series to validate the simulation results. Since the new optrode setup without lens and with elongated fiber guide length has not been fabricated yet only the effect of the tip shape has been investigated by measurements. The measurements were taken for optrodes with rectangular shape tip as well as $15^{\circ}$ angle symmetric tip. 
The view of the optrode in optical measurement setup can be seen on Fig. 9.

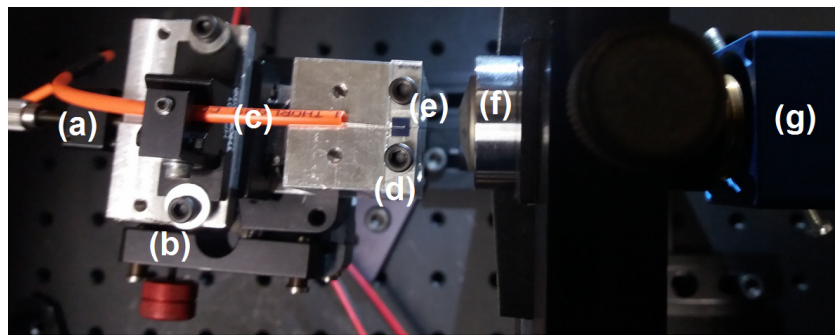

Fig.9. Top view of the optrode in an optical measurement setup (a) 1D translation stage to set the position of the fiber (b) 3D translation stage (c) multimode fiber (d) sample holder (e) optrode with $15^{\circ}$ angle symmetric tip (f) microscope objective $(50 \mathrm{x}, \mathrm{NA}=0.8)(\mathrm{g})$ CMOS beam profiler.

We have investigated the spatial distribution of the output light using a CMOS camera, and we have observed a qualitative agreement between the simulated and measured light field as shown on Fig.10.
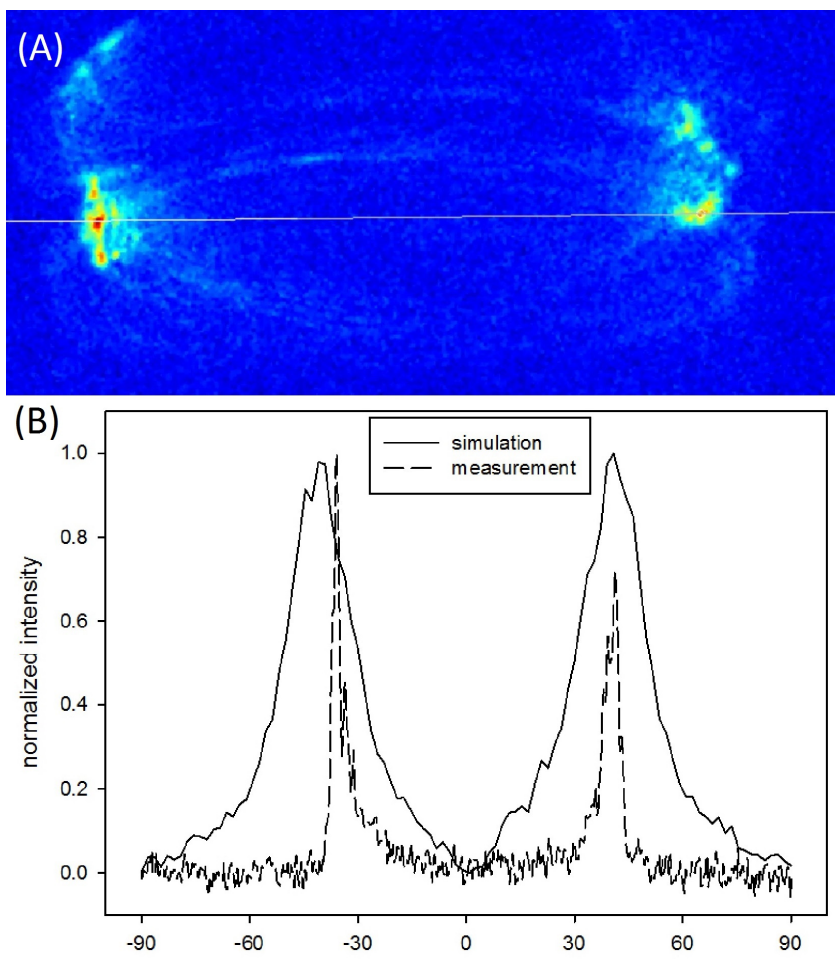

Fig.10. (A) Measured spatial distribution of the output light (B) Comparison of the measured cross-section along a line in (A) and the simulation results

The observable asymmetry of the experimental curve is due to the fabrication imperfections of the investigated optrode device.

\section{CONCLUSION}

Simulation results have been presented to optimize a silicon optrode regarding the mechanical strength, optical device efficiency and temperature rise of the brain tissue during near-infrared stimulation. Eliminating the lens and elongating the fiber guide length by 0.94 $\mathrm{mm}$ conserved the mechanical strength of the optrode and led to $33 \%$ relative improvement of the overall device efficiency due to better in-coupling into the shaft. Changing the tip from rectangular shape to $15^{\circ}$ angle sharp tip decreased the reflection at the silicon-air boundary therefore led to further $17 \%$ relative improvement of the device efficiency. Using an excitation of $30 \mathrm{~mW}$ peak power and 100 ms pulse width, the value of the maximal temperature rise occurred within $0.1-0.2 \mathrm{~mm}$ from the tip with the value of $4.42^{\circ} \mathrm{C}$ instead of 3 ${ }^{\circ} \mathrm{C}$ belonging to the original setup. Our simulation results serve as a guideline to develop optrode devices for near-infrared brain stimulation with optimized mechanical, optical and thermal properties.

Funding. National Brain Research Program (2017_1.2.1-NKP-201700002); European Union (EFOP-3.6.3-VEKOP-16-2017-00002); New National Excellence Foundation (UNKP-18-4-PPKE-2; UNKP18-3-I-OE-90)

\section{References}

1. J. Wells, C. Kao, P. Konrad, T. Milner, J. Kim, A. Mahadevan-Jansen, E.D. Jansen, "Biophysical mechanisms of transient optical stimulation of peripheral nerve," Biophys. J. 93, 2567-2580 (2007).

2. M. M. Chernov, G. Chen, A. W. Roe "Histological Assessment of Thermal Damage in the Brain Following Infrared Neural Stimulation," Brain Stimulation 7, 476-482 (2014).

3. J. M. Cayce, R.M. Friedman, E.D. Jansen, A. Mahavaden-Jansen, A.W. Roe "Pulsed infrared light alters neural activity in rat somatosensory cortex in vivo," Neuroimage 57, 155-166 (2011).

4. Á. C. Horváth, Ö. C. Boros, S. Beleznai, Ö. Sepsi, P. Koppa, Z. Fekete “A multimodal optrode for spatially controlled infrared neural stimulation in the deep brain tissue," Sens.Actuators B-Chem. 263, 77-86 (2018).

5. Ö. C. Boros, Á. C. Horváth, S. Beleznai, Ö. Sepsi, S. Lenk, Z. Fekete, P. Koppa "Optical and thermal modeling of an optrode microdevice for infrared neural stimulation," App.Opt. 57, 6952-6957 (2018).

6. F. -J. Haug, M. Brauninger, C. Ballif "Fourier light scattering model for treating textures deeper than the wavelength," Opt. Express 25(4), A14A22 (2017).

7. F. J. Haug, C. Battaglia, D. Domine, and C. Ballif "Light scattering at nanotextured surfaces in thin film silicon solar cells," IEEE PVSC 35, 754-759 (2010).

8. R. Liljemalm, T. Nyberg, H. von Holst "Heating during infrared neural Stimulation," Lasers Surg. Med. 45, 469-481 (2013).

9. T. V. F. Abaya, M. Diwekar, S. Blair, P. Tathireddy, L. Rieth, F. Solzbachera "Deep-tissue light delivery via optrode arrays," Biomed Opt. 19(1), 015006 (2014).

10. S. Dufour, P. Dufour, F. Amzica, R. Vallée "Neurobiological use of a micro-optrode using UV excitation light and signal-to-noise ratio optimization," Proc. of SPIE Vol. 7161, 1-8 (2009).

11. M. Giaquinto, A. Micco, A. Aliberti, E. Bobeico, V. La Ferrara, R. Menotti, A. Ricciard, A. Cusano "Optimization Strategies for Responsivity Control of Microgel Assisted Lab-On-Fiber Optrodes," Sensors 18, 1119 (2018).

12. https://www.comsol.com/comsol-multiphysics

13. Comsol Structural Mechanics Module Users Guide, page 105.

14. L. Zhang, R. Barrett, P. Cloetens, C. Detlefs and M. Sanchez del Rio "Anisotropic elasticity of silicon and its application to the modelling of $X$ ray optics," J. Synchrotron Rad. 21, 507-517 (2014).

15. R. von Mises "Mechanik der festen Körper im plastisch deformablen Zustand," Göttin. Nachr. Math. Phys. 1, 582-592 (1913).

16. Zemax ${ }^{\circledR}$ User's manual, https://www.zemax.com

17. Z. Fekete, A. Németh, G. Márton, I. Ulbert, A. Pongrácz "Experimental study on the mechanical interaction between silicon neural microprobes and rat dura mater during insertion," J. Mater. Sci.: Mater. Med. 26, 70-79 (2015).

18. M. Kiss, P. Földesy, Z. Fekete "Optimization of a Michigan-type silicon microprobe for infrared neural stimulation," Sens.Actuators B-Chem. 224, 676-682 (2016) 
